\title{
Embedding an Arbitrary 1-safe Petri Net into a Boolean Petri Net
}

\author{
Gajendra Pratap Singh \\ Department of Applied Mathematics \\ Delhi Technological University \\ Shahbad Daulatpur, Main Bawana Road \\ Delhi-110042, India
}

\author{
Sangita Kansal \\ Department of Applied Mathematics \\ Delhi Technological University \\ Shahbad Daulatpur, Main Bawana Road \\ Delhi-110042, India
}

\author{
Mukti Acharya \\ Department of Applied Mathematics \\ Delhi Technological University \\ Shahbad Daulatpur, Main Bawana Road \\ Delhi-110042, India
}

\begin{abstract}
Petri nets are powerful mathematical formalism for designing and studying behaviors of a wide range of discrete dynamical event driven systems. The aim of this paper is to show that an arbitrary 1safe Petri net can be embedded as an induced subnet of a Boolean Petri net, viz., the one that generates every binary $n$-vector as its marking vector.
\end{abstract}

\section{Keywords:}

1-safe Boolean Petri net, reachability tree, binary $n$-vector, marking vector.

\section{INTRODUCTION}

A Petri net is a graphical tool invented by Carl Adam Petri [1]. Its origin can be traced back to August 1939 when, at the age of 13 , Petri created the graphics to describe chemical processes that produce a final compound from various elements through some intermediate compounds. The 'net-like' representation of these logical tools came into the existence in his doctoral thesis "Communication with Automata" at the Technical University of Darmstadt, Germany, in 1962 [1]. Since then Petri nets have been developed and used in many theoretical as well as application areas [2] 3, 4, 5]. A Petri net may be identified as a particular kind of bipartite directed graph having three types of objects. These objects are places, transitions and directed arcs connecting places to transitions and transitions to places. Pictorially, places are depicted by circles and transitions as bars or boxes. A place is an input place to a transition if there exists a directed arc connecting this place to the transition. A place is an output place of a transition if there exists a directed arc connecting the transition to the place. Places and transitions may have many interpretations and for other meanings for places and transitions the reader is referred to [6]. Although some structural techniques [7] have been successfully developed to study the 1-safe systems, unfortunately, they often offer only semi decision conditions, i.e., either necessary or sufficient conditions, or their application is restricted to some specific subclasses of Petri nets. Keeping this drawback in mind, the authors (cf.: Kansal et al. [8]) proposed a 1-safe star Petri net $S_{n}$, with $|P|=n$ and $|T|=n+1$, having a central transition, that generates all the binary $n$-vectors, as its marking vectors; they also established the existence of 1-safe Petri nets that generate all the binary $n$-vectors exactly once as marking vectors.

\section{PRELIMINARIES}

For standard terminology and notation on Petri net theory and graph theory, we refer the reader to Peterson [7] and Harary [9]. In this paper, we shall adopt the definition of Jenson [10]:

A Petri net is a 5 -tuple $N=\left(P, T, I^{-}, I^{+}, \mu^{0}\right)$, where

(1) $P$ is a nonempty set of 'places',

(2) $T$ is a nonempty set of 'transitions',

(3) $P \cap T=\emptyset$,

(4) $I^{-}, I^{+}: P \times T \longrightarrow \mathbf{N}$, where $\mathbf{N}$ is the set of nonnegative integers, are called the negative and the positive 'incidence functions' (or, 'flow functions') respectively,

(5) $\forall p \in P, \exists t \in T: I^{-}(p, t) \neq 0$ or $I^{+}(p, t) \neq 0$ and $\forall t \in T, \exists p \in P: I^{-}(p, t) \neq 0$ or $I^{+}(p, t) \neq 0$,

(6) $\mu^{0}: P \rightarrow \mathbf{N}$ is the initial marking.

In fact, $I^{-}(p, t)$ and $I^{+}(p, t)$ represent the number of arcs from $p$ to $t$ and $t$ to $p$ respectively. $I^{-}, I^{+}$and $\mu^{0}$ can be viewed as matrices of size $|P| \times|T|,|P| \times|T|$ and $|P| \times 1$, respectively.

As in many standard books (e.g., see [11]), Petri net is a particular kind of directed graph, together with an initial marking $\mu^{0}$. The underlying graph of a Petri net is a directed, weighted, bipartite graph consisting of two kinds of nodes, called places and transitions, where arcs are either from a place to a transition or from a transition to a place.

The initial marking is represented by placing a token in the circle representing a place $p_{i}$ as a black dot whenever $\mu^{0}\left(p_{i}\right)=1$, $1 \leq i \leq n=|P|$. In general, a marking $\mu$ is a mapping $\mu: P \longrightarrow \mathbf{N}$. A marking $\mu$ can hence be represented as a vector $\mu \in \mathbf{N}^{n}, n=|P|$, such that the $i^{t h}$ component of $\mu$ is the value $\mu\left(p_{i}\right)$.

Let $N=\left(P, T, I^{-}, I^{+}, \mu\right)$ be a Petri net. A transition $t \in T$ is said to be enabled at $\mu$ if and only if $I^{-}(p, t) \leq \mu(p), \forall p \in$ 
$P$. An enabled transition may or may not 'fire' (depending on whether or not the event actually takes place). After firing at $\mu$, the new marking $\mu^{\prime}$ is given by the rule

$$
\mu^{\prime}(p)=\mu(p)-I^{-}(p, t)+I^{+}(p, t), \text { forall } p \in P
$$

We say that $t$ fires at $\mu$ to yield $\mu^{\prime}$ (or, that $t$ fires $\mu$ to $\mu^{\prime}$ ), and we write $\mu \stackrel{t}{\longrightarrow} \mu^{\prime}$, whence $\mu^{\prime}$ is said to be directly reachable from $\mu$. Hence, it is clear, what is meant by a sequence like

$$
\mu^{0} \stackrel{t_{1}}{\longrightarrow} \mu^{1} \stackrel{t_{2}}{\longrightarrow} \mu^{2} \stackrel{t_{3}}{\longrightarrow} \mu^{3} \cdots \stackrel{t_{k}}{\longrightarrow} \mu^{k}
$$

which simply represents the fact that the transitions $t_{1}, t_{2}, t_{3}, \cdots, t_{k}$ have been successively fired to transform the marking $\mu^{0}$ into the marking $\mu^{k}$. The whole of this sequence of transformations is also written in short as $\mu^{0} \stackrel{\sigma}{\longrightarrow} \mu^{k}$, where $\sigma=t_{1}, t_{2}, t_{3}, \cdots, t_{k}$ denotes the corresponding firing sequence.

DEFINITION 1. A marking $\mu$ is said to be reachable from $\mu^{0}$, if there exists a sequence of transitions which can be successively fired to obtain $\mu$ from $\mu^{0}$. The set of all markings of a Petri net $N$ reachable from a given marking $\mu$ is denoted by $\mathcal{M}(N, \mu)$ and, together with the arcs of the form $\mu^{i} \stackrel{t_{r}}{\longrightarrow} \mu^{j}$, represents what in standard terminology is called the reachability graph of the Petri net $N$. If the reachability graph has no cycle then it is called reachability tree.

It has been found and established that the reachability tree of a 1-safe Petri net can be homomorphically mappped on to the $n$-dimensional complete Boolean lattice, thereby yielding new techniques to represent the dynamics of these Petri nets [12].

DEFINITION 2. A place in a Petri net is 1-safe if the number of tokens in that place never exceeds one. A Petri net is 1-safe if all its places are 1-safe.

Note that there is another way to represent Petri net elements as given in the following definition.

DEFINITION 3. The preset of a transition $t$ is the set of all input places to $t$, i.e., ${ }^{\bullet} t=\left\{p \in P: I^{-}(p, t)>0\right\}$. The postset of $t$ is the set of all output places from $t$, i.e., $t^{\bullet}=$ $\left\{p \in P: I^{+}(p, t)>0\right\}$. Similarly, $p$ 's preset and postset are - $p=\left\{t \in T: I^{+}(p, t)>0\right\}$ and $p^{\bullet}=\left\{t \in T: I^{-}(p, t)>0\right\}$, respectively.

\section{MAIN RESULT}

In this section we will give the theorem for embedding a 1-safe Petri net into a Boolean Petri net. This theorem indicates that one cannot hope to have a forbidden subgraph characterization of a Boolean Petri net.

THEOREM 4. Every 1-safe Petri net $N=$ $\left(P, T, I^{-}, I^{+}, \mu^{0}\right),|P|=n$ with $\mu^{0}(p)=1 \quad \forall p \in P$ can be embedded as an induced subnet of a Boolean Petri net.

Proof. Let $N=\left(P, T, I^{-}, I^{+}, \mu^{0}\right),|P|=n$ be a 1 -safe Petri net. If $N$ is Boolean Petri net then there is nothing to prove. Hence, assume that $N$ is not a Boolean Petri net. Then, we have the following steps to obtain a Boolean Petri net $N^{\prime}$ in which $N$ is one of its induced subnets.

Step-1: First of all, find those places in $N$ whose postsets have single distinct sink transitions (if the postset of a place has more than one distinct sink transitions then choose only one transition giving $K_{2}$ ). Suppose such places are $p_{1}, p_{2}, \cdots, p_{k}, 0 \leq k<n$. Step-2: Augment $n-k$ new transitions and join each transition to the remaining $n-k$ places in $N$ by an arc from a place to a transition creating $n-k$ new active transitions.
Step-3: Thus, in $N^{\prime}$ we have $n$-copies of $K_{2}$ as its subgraph. Since $\mu^{0}(p)=1 \forall p \in P$, all the transitions are enabled. Firing of $n$ transitions forming $n$ 'pendant transitions' will produce ${ }^{n} C_{1}$ distinct binary $n$-vectors whose Hamming distance is 1 from the initial marking vector. At these marking vectors, $n-1$ transitions out of those $n$ transitions are enabled, and after firing give at least ${ }^{n} C_{2}$ distinct marking vectors, each of whose Hamming distance is 2 from the initial marking.

In general at any stage $j, 3 \leq j \leq n$, we get a set of at least ${ }^{n} C_{j}$ new distinct binary $n$-vectors whose Hamming distance is $j$ from the initial marking, which are also distinct from the sets of ${ }^{n} C_{r}$ distinct marking vectors for all $r, 2 \leq r \leq j-1$. Therefore, at the $n^{t h}$ stage we would have obtained at least ${ }^{n} C_{1}+{ }^{n} C_{2}+\cdots+{ }^{n} C_{n}=2^{n}-1$ distinct binary $n$-vectors. Together with the initial marking $(1,1, \cdots, 1)$, we thus see that all the $2^{n}$ binary $n$-vectors would have been obtained as marking vectors, possibly with repetitions. Thus $N^{\prime}$ is Boolean.

Therefore, every 1-safe Petri net $N$ can be embedded as an induced subnet of a Boolean Petri net.

The above theorem demonstrates indirectly that the problem of deciding whether a given 1-safe Petri net is Boolean is an NPcomplete problem. Therefore, it becomes important to look for specific classes of 1-safe Petri nets having computationally good characterization would entirely depend upon whether a particular class is theoretically interesting or practically essential to have. The extent and effectiveness of embedding utility in solving the practical problem requiring the design of multi-functional switches for the operation of certain discrete dynamical systems of common use as washing machines and teleprinters [13]. One immediate class that comes to our mind in this context is that of the class of crisp Boolean Petri nets, viz, a 1-safe Petri net that generates every binary $n$-vector as their marking vector exactly once. Existence and uniqueness of crisp Boolean Petri net has been reported in [14 15]. We strongly believe that even this class may not a forbidden subgraph characterization.

\section{REFERENCES}

[1] Petri, C.A., Kommunikation mit automaten, Schriften des Institutes fur Instrumentelle Mathematik, Bonn, 1962.

[2] Callou, G., Macial, P., Tutsch, D., Araujo, J., Ferreira, J. and Souza, R., A Petri net-based approach to the quantification of data center dependability. In: Petri nets - Manufacturing and Computer Science (Ed.: Pawel Pawlewski), 2012, 313-336.

[3] Dassow, J., Mavlankulov, G., Othman, M., Turaev, S., Selamat, M.H. and Stiebe, R., Grammers controlled by Petri nets. In: Petri nets - Manufacturing and Computer Science (Ed.: Pawel Pawlewski), 2012, 337-358.

[4] Lee, G.B., Zandong, H. and Lee, J.S., Reachability criterion with sufficient test space for ordinary Petri net. In: Petri nets - Manufacturing and Computer Science (Ed.: Pawel Pawlewski), 2012, 423-438.

[5] Lee, G.B., Zandong, H. and Lee, J.S., Generalized state equation of Petri nets with priority, International Journal of Intelligent Systems, 18(11), 2003, 1145-1153.

[6] Murata, T., Petri nets: Properties, analysis and applications, Proc. IEEE, 77(4), 1989, 541-580.

[7] Peterson, J.L., Petri net Theory and the Modeling of Systems, Englewood Cliffs, NJ: Prentice-Hall, Inc., 1981.

[8] Kansal, S., Singh, G.P., Acharya, M., On Petri nets generating all the binary $n$-vectors, Scientiae Mathematicae Japonicae, 71(2)2010, 209-216.

[9] Harary, F., Graph Theory, Addison-Wesley, Massachusettes, Reading, 1969. 
[10] Jensen, K., Coloured Petri nets, Lecture notes in computer Science, 254, Springer-Verlag, Berlin, 1986, 248-299.

[11] Reisig, W., Petri nets, Springer-Verleg, New York, 1985.

[12] Kansal, S., Singh, G.P. and Acharya, M., A disconnected 1-safe Petri net whose reachability tree is homomorphic to a complete boolean lattice, Proceeding of International Conference on Process Automation Control and Computing, IEEE Xplore, 2011, 1-5, ISBN : 978-1-61284-762-7.

[13] Acharya, B.D., Set-indexers of a graph and set-graceful graphs, Bull. Allahabad Math. Soc., 16, 2001, 1-23.

[14] Kansal, S., Singh, G.P., Acharya, M., 1-Safe Petri nets generating every binary $n-v e c t o r$ exactly once, Scientiae Mathematicae Japonicae, 74(1), 2011, 29-36.

[15] Kansal, S., Acharya, M. and Singh, G. P., Uniqueness of minimal 1 -safe Petri net generating every binary $n$-vectors as its marking vectors exactly once, Scientiae Mathematicae Japonicae, 74(2 \& 3), 2011, 117-120. 